\title{
On the limits of perceptual complementarity in the kinetic depth effect
}

\author{
TERRY CAELLI, PATRICK FLANAGAN, and STEPHEN GREEN \\ University of Newcastle, Newcastle, N.S. W., Australia
}

\begin{abstract}
In a series of experiments, we have investigated the abilities of human observers to perceive geometric properties of moving three-dimensional objects as a function of their perspective and rotational complexities. The results indicate a decreasing ability of observers to extract metric, angular, and rigid motion as the perspectives and rotations depart from parallel projections and one-parameter central rotations. In this way, quantitative limits are suggested for the principle of perceptual complementarity suggested by Shepard (1981).
\end{abstract}

From the earlier observations of Wallach and O'Connell (1953) on the kinetic depth effect (KDE) to more recent demonstrations by Shepard and his associates (see Shepard, 1981, for a review), it is clear that one can extract the percept of a rigid threedimensional (3D) rotating object from a sequence of two-dimensional (2D) perspective views when the spatiotemporal display parameters are adequate for apparent motion to occur. Such results have led Shepard (1981) to propose a principle of "complementarity" whereby percepts are argued to reflect the nature of the three-dimensional spatial world where objects are invariant under rigid motions. However, to this stage, the specific parametric determinants of this 2D-to-3D coding process have not been systematically studied.

To reduce the problem to its simplest form, we wish to know under what conditions the visual system can enact the inverse of the general projection equation:

$$
\left[\begin{array}{l}
x^{\prime}(t) \\
y^{\prime}(t)
\end{array}\right]=(r-f)\left[\begin{array}{l}
x(t) /[r-z(t)] \\
y(t) /[r-z(t)]
\end{array}\right]
$$

where $\{x(t), y(t), z(t)\}$ corresponds to a 3D motion, and $\left\{x^{\prime}(t), y^{\prime}(t)\right\}$, its $2 D$ projection. Here $(r, f)$ refer to the viewing and projection plane distances from the origins (Figure 1). Also, the transformations $\{x(t), y(t)$, $z(t)\}$ are considered to assume the general linear form

$$
\begin{aligned}
& x(t)=a_{1}(t) \cos \theta(t) \cdot \sin \phi(t)+a_{2}(t) \\
& y(t)=b_{1}(t) \cos \theta(t) \cdot \cos \phi(t)+b_{2}(t) \\
& z(t)=c_{1}(t) \sin \theta(t)+c_{2}(t)
\end{aligned}
$$

The authors' complete address is: Department of Psychology, University of Newcastle, Newcastle, N.S.W. 2308, Australia. for rotation angles $\theta(\mathrm{t}), \phi(\mathrm{t})$ about the $\mathrm{z}$ and $\mathrm{x}$ axes, respectively ${ }^{1}$ (Figure 1$),\left\{a_{i}(t), b_{i}(t), c_{i}(t)\right\}$ being linear transformation coefficients.

By defining the "3D reconstruction" problem (2Dto-3D) as one of perceptually deducing $\{x, y, z\}$ from $\left\{x^{\prime}, y^{\prime}\right\}$, it is clear (Equation 1) that, even if we correctly code the perspective parameters $(r, f)$, the problem cannot be uniquely solved with one perspective view. However, it can be solved when more than one frame is taken-for example, when we have motion or at least two perspective views. In this latter case, it is easily shown that, from the two sets of observations involving finite $r$ and $f$ values (infinite $r$ corresponds to parallel projections),

$$
\left\{\left(\mathrm{x}_{1}, \mathrm{y}_{1} ; \mathrm{r}_{1}, \mathrm{f}_{1}\right),\left(\mathrm{x}_{2}, \mathrm{y}_{2} ; \mathrm{r}_{2}, \mathrm{f}_{2}\right)\right\},
$$

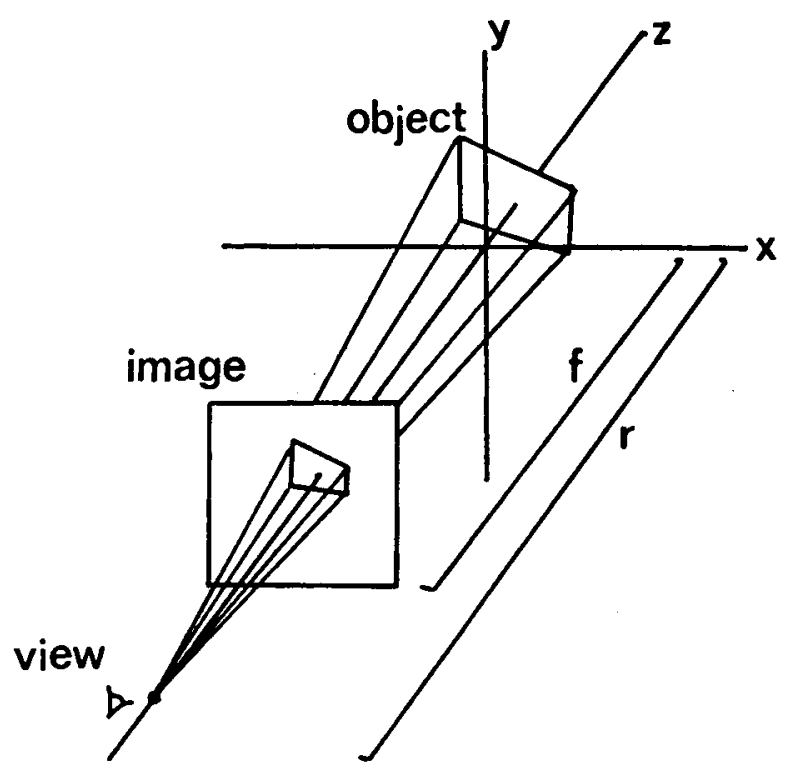

Figure 1. Definition of perspective parameters: $r$ is the viewing distance and $f$, the projection plane distance from a given origin. 
$(\mathrm{x}, \mathrm{y}, \mathrm{z})$ are determined by

$$
\begin{aligned}
& x=\frac{x_{1}\left(r_{1}-z\right)}{r_{1}-f_{1}} \\
& y=\frac{y_{1}\left(r_{1}-z\right)}{r_{1}-f_{1}} \\
& z=\frac{\left(r_{2}-f_{2}\right) x_{1} r_{1}-\left(r_{1}-f_{1}\right) x_{2} r_{2}}{\left(r_{2}-f_{2}\right) x_{1}-\left(r_{1}-f_{1}\right) x_{2}} .
\end{aligned}
$$

It is clear from this example that the $3 \mathrm{D}$ reconstruction process is dependent on how the $(r, f)$ projection constants are registered, such that if the observer does not veridically code these distances a correct reconstruction would not be possible. Other limitations in the reconstruction process may include temporal frequency and the spatiotemporal complexity of the images (Caelli, 1981a). For example, Braden (1978) has shown that the amount of linear perspective is a strong determinant of perceived (3D) oscillations with rotating trapezoidal windows. Equally, Caelli $(1980,1981 \mathrm{~b})$ has also shown that the visual system cannot fully process curvature or torsion information in rotating $3 \mathrm{D}$ objects. Examples of spatiotemporal limitations include the fact that the KDE is limited to temporal frequencies (of rotation) below $2 \mathrm{~Hz}$ and the fact that it exhibits a phase sensitivity curve symmetrical about $90 \mathrm{deg}^{2}$ (Caelli, 1980, 1981b). The point of these examples is that there are many event configurations which do prohibit the extraction of $3 \mathrm{D}$ objects by the human observer, so delimiting the existence conditions for "perceptual complementarity."

The aim of the following experiments was to systematically examine (under a variety of conditions and tasks) the perspective and rotational determinants of the reconstruction process in such a way that the underlying "perceptual geometry" could be defined.

To simplify matters, linear perspective may be defined as a number between 0 and 1 , determined by the ratio of the furthest/nearest possible extent changes as a function of the $(r, f)$ values (viewing distance and projection plane distance from an object centered at the origin). Since the projection extent along both $\mathrm{x}$ and $y$ axes is determined by Equation 1 as $(r-f) /$ $(r-z)$ and the limiting depth values are $z= \pm f$, the linear perspective, $L_{p}$, is:

$$
0<L_{p}=\frac{r-f}{r+f}<1
$$

In reality, $L_{p}$ cannot be 1 or 0 due to the fact that

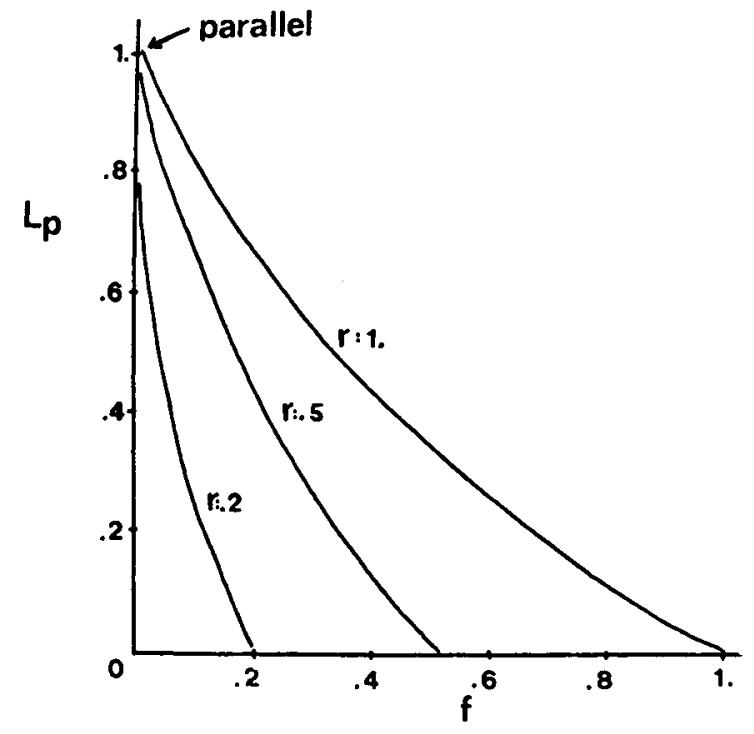

Figure 2. The relationship between linear perspective $\left(L_{p}\right)$ and $(r, f)$ conditions illustrated in Figure 1.

these extreme values are the limiting cases of parallel projections (observer's view is at infinity: $r=\infty, L_{p}=$ 1.0 ) and "infinite perspective" (the observer's viewing position is at the projection plane: $r=f, L_{p}=0$ ). However, the measure does index perspective between these two extremes, as shown in Figure 2.

This relationship between linear perspective and projection distances implies that a necessary condition for the ability to reconstruct a 3D object from its projection is the veridical coding of the $r$ and $f$ parameters. So, in the first experiment to be reported, observers were asked to estimate their viewing distance from the center of a rotating object and the projection plane as a function of various perspective views.

\section{EXPERIMENT 1: ON PERCEIVED (r,f) VALUES}

\section{Method}

Subjects. Seven undergraduate students from the University of Newcastle were used (all volunteers). None had a history of visual defects.

Stimulus and Apparatus. As already indicated, previous studies of perceived rotations usually employ parallel projections with the observer (theoretical) positioned at an infinite distance from the source (Figure 1). The role of linear perspective has been raised in only a few studies (e.g., Braden, 1978). On the other hand, size cues for depth perception have been extensively investigated both with regard to magnitude estimation psychophysics (e.g., Gilinsky, 1951) and size constancy (Robinson, 1972). In a similar way to the determination of linear perspective, size modulation may be determined by:

$$
L_{s}=1-\frac{f}{r} .
$$

Conversely, both size and linear perspective information determine $r$ and $f$ values. 
These experiments were run entirely on a computer-driven (PDP$11 / 40$ ) display (HP 1310A, P15 phosphor) coupled to a HP 1350 graphics translator, so enabling real-time computer-generated rotating (or static) objects (Figure 3).

$A$ cube and projection frame were computer generated and displayed onto the CRT screen for the following $(r, f)$ values (Figure 3):

$$
\begin{aligned}
\mathrm{I} & =550,1,100,2,200,4,400 \text { (display units) } \\
& =13.75,27,55,108 \mathrm{~cm} \\
\mathrm{f} & =300,400,500 \text { (display units) } \\
& =7.5,10,13.5 \mathrm{~cm} .
\end{aligned}
$$

Three different presentation conditions were employed: (1) the image was statically presented for $4 \mathrm{sec}$ from a "front-on" view; (2) the cube was set rotating about the y-axis alone at $1 \mathrm{~Hz}$; and (3) the cube was set rotating about the $x$ - and $y$-axes simultaneously. These three conditions were used to investigate whether the amount of dynamic change in the image induced more stable and accurate percepts of $3 D$ rotations and, so, depth information.

Although different $r$ values were used, subjects were always seated $1 \mathrm{~m}$ from the screen and viewed the CRT monocularly with their dominant eye (Figure 3). Space average luminance of the stimulus was $12 \mathrm{~cd} / \mathrm{m}^{2}$.

Procedure. The subjects were given a brief verbal description of the task and told that the experiment was concerned with their ability to judge distances. The stimulus was described as a 4 -in. cube (all subjects preferred imperial measurements) with a small centered dot. They were also told that the projection plane reference square was $6 \times 6$ in. On any one trial, the subject was to judge the distance (feet and inches) between themselves and the cube center, themselves and the projection plane, or the projection plane and the cube center (this latter response was not used in the analysis). The only other limitation imposed was that they were told the total apparatus length $(8 \mathrm{ft}$ from viewing position to the end; Figure 3). The whole device was covered by a black cloth material to prohibit subjects from seeing where the actual CRT screen was positioned.
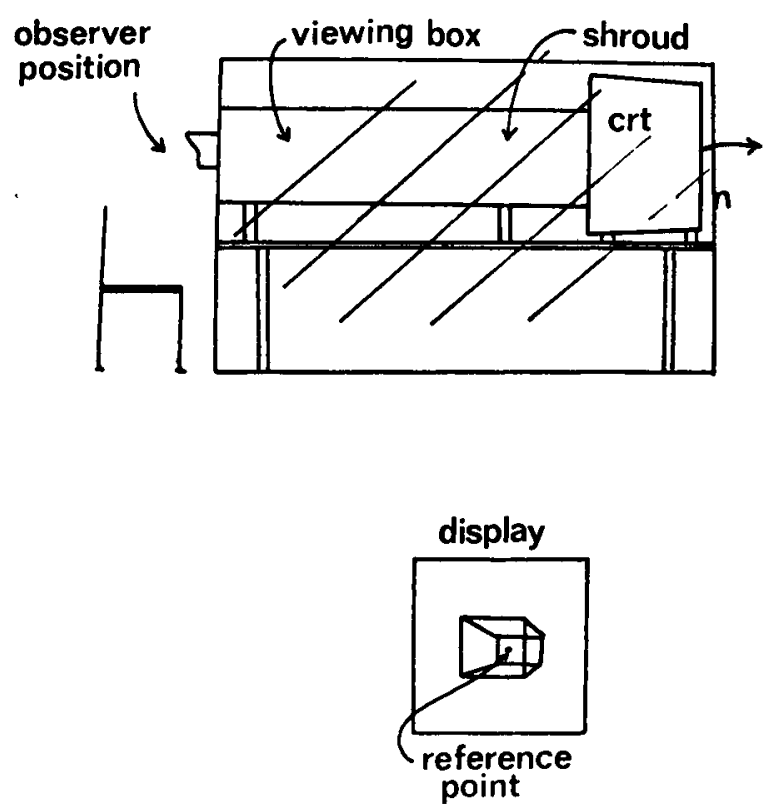

Figure 3. Viewing box and display conditions for Experiments 1,2 , and 3.
An experimental trial consisted of the presentation of one $(r, f)$ combination with only one of the distance responses defined above. For each condition, the subjects received eight replications. Each stimulus was exposed for $4 \mathrm{sec}$, after which they were asked to respond as quickly and as accurately as possible. The subjects were asked to fixate on the reference point for all trials, and all observations were made monocularly with the dominant eye.

\section{Results}

Figure 4 illustrates the average perceived distances for each judgment as a function of the size, perspective, and rotation type.

Since no detectable differences were present in these data as a function of the dynamic/static presentation conditions, and within- and between-subject variations were very small for both sets of distance estimates (Figure 4), a multiple regression was computed on the distance judgments ( $\psi_{\mathrm{d}}$ : subject to cube center) as a function of the size $\left(\mathrm{L}_{\mathrm{s}}\right)$ and perspective $\left(L_{p}\right)$ parameters resulting in

$$
\psi_{\mathrm{d}}=-88.05 \mathrm{~L}_{\mathrm{s}}+52.63 \mathrm{~L}_{\mathrm{p}}+73.27
$$

which explains $87 \%$ of the total variance $-54 \%$ for size and $33 \%$ for perspective $(R=.93, p<.01)$. It should be noted that, although distance judgments were linearly related to size and perspective parameters, and varied monotonically with the specified $r$,f values (Figure 4), the absolute distances were generally overestimated. For example, when $r$ was set at 44 in. $(110 \mathrm{~cm})$ and $\mathrm{f}$ at 5 in. $(12.5 \mathrm{~cm})$, the judged distance was almost twice the $r$ value. Interestingly, as perspective increased ( $\mathrm{r}$ decreased), the distance estimates became more accurate.

We can conclude from these results that, for relatively short distances (short in comparison with those studied by Gilinsky, 1951, for example), we are capable of adopting reference frames, other than the observer's physical position, to judge projected distances of objects as a function of the simplest of cues: size and perspective. In all conditions, observers' estimates of $(r, f)$ values vary monotonically with objective $(r, f)$ values, although an absolute correspondence was not found.

These results indicate that the perspective and size information portrayed in the $2 \mathrm{D}$ image provides not only conditions of the underlying $3 \mathrm{D}$ image, but also metric information about the observer's position and projection plane. Specifically, with adult observers the reference frame (defined by $r, f$ ) need not be egocentric, but only internally consistent (see Caelli, Hoffman, \& Lindman, 1978, for further discussion on the relativity of reference frames in motion).

The reconstruction process was defined as the ability to enact the inverse of Equation 1, and this process is critically dependent on the $(r, f)$ states. The point of this first experiment is that we have found that perceived $(r, f)$ values are determined by perspective and size parameters of the projected image. This, 

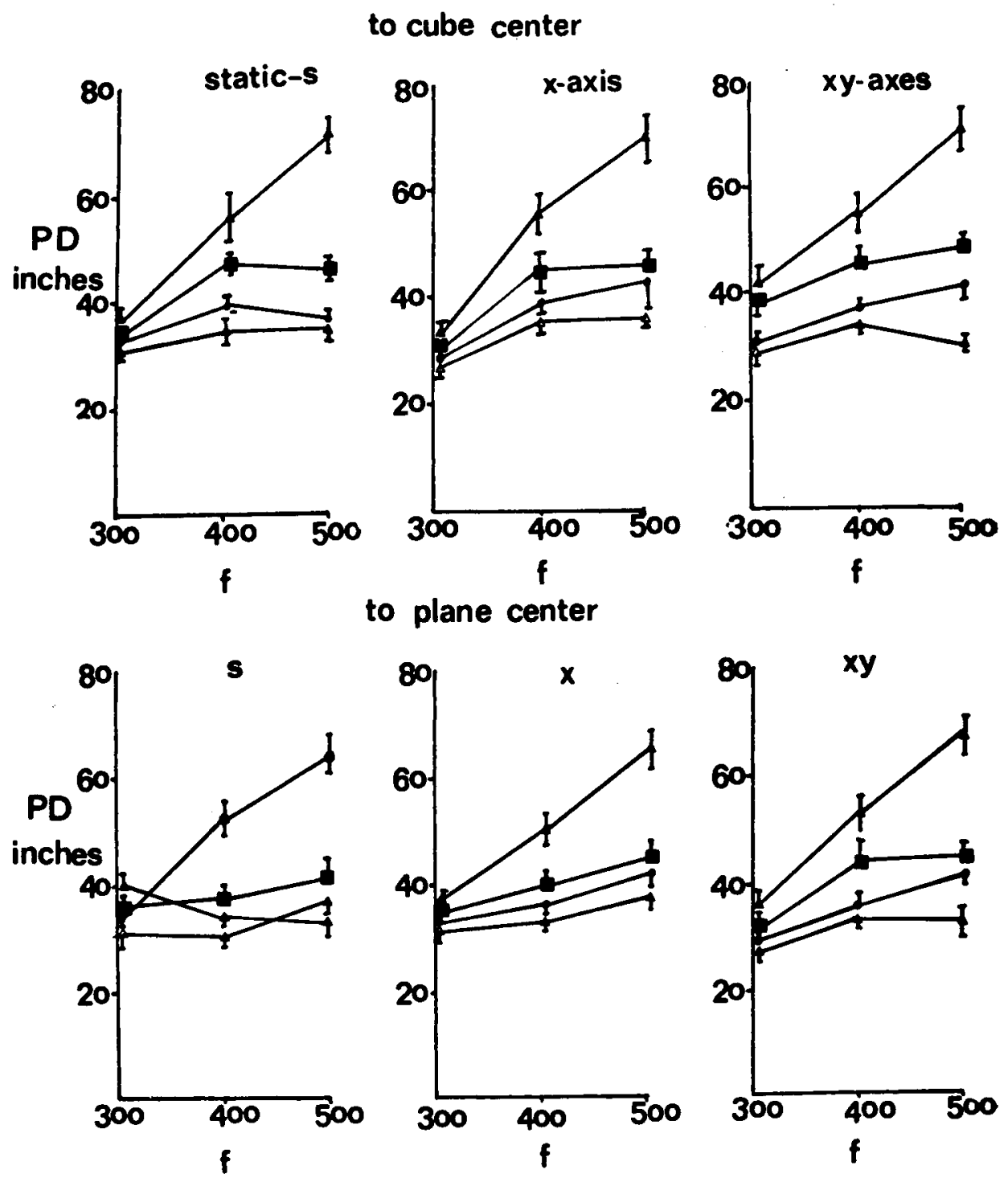

Figure 4. Perceived distance (PD) to center point and plane as a function of $(r, f)$ values and rotation type (PD in inches): $(r, f)$ in display units (1 unit =.01 in.) $\Delta, r=550 ; 0, r=1,100 ; \bullet, r=2,200$; and $\Delta, r=4,400$ (bottom curve in each graph).

in turn, suggests that observers may well use estimated $(r, f)$ states to determine the 3D status of the projected image. In the following experiment, the aim was to investigate whether very primitive geometric properties of the subjectively reconstructed 3D image are euclidean in nature and determined by the $(r, f)$ reference frame and rotation information.

\section{EXPERIMENT 2: \\ PROJECTION DETERMINANTS OF PERCEIVED LENGTH AND ANGLE}

Perhaps the two most important geometric properties of objects are lengths and angles. It is also clear that in order to perceive rigid motions of 3D objects, perceived angles and lengths must be preserved. This is the nature of euclidean geometry and motions.
Affine transformations (the geometry of perspectives; Caelli, 1981a), however, do not preserve lengths but, rather, simply preserve angles. So far we have simply demonstrated that observers have an "internal perspective view" (or reference frame) consistent with the perspectivities involved in the $2 \mathrm{D}$ projections. We have not explored the conditions under which this situation allows the reconstruction of invariant angles and/or lengths. Such conditions further define the limits of perceptual complementarity.

\section{Method}

Subjects. Six undergraduate students from the University of Newcastle were used (all volunteers) having no history of visual defects. (No common subjects were used in all the experiments reported.)

Stimulus and Apparatus. Stimuli were all generated on the PDP-11/40-HP graphics translator system and displayed on the 
line
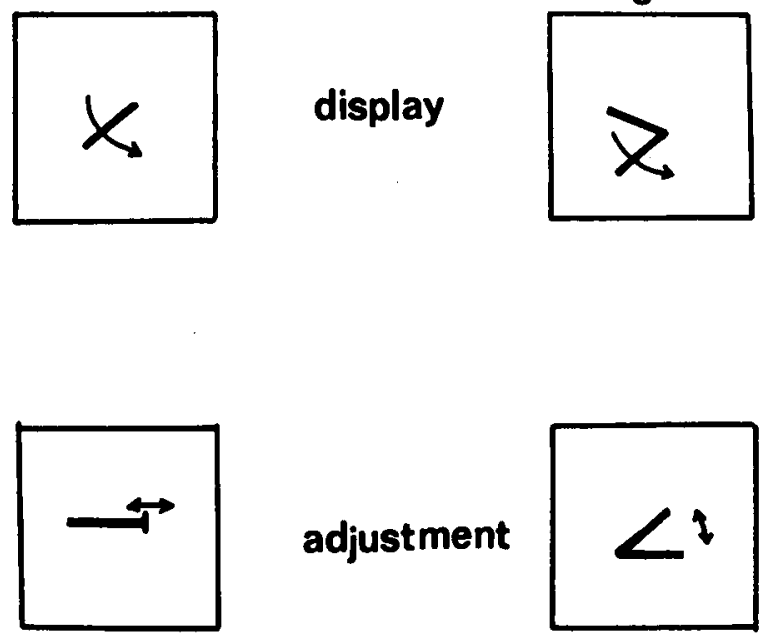

Figure 5. Stimulus types used in Experiment 2, including response apparatus.

HP 1310A CRT. Figure 5 illustrates the two stimulus types employed: single-line and double-line-angle cases. These simple stimuli were used such that the basic parameters of length and angle could be examined in isolation. This is not to imply that, with more complex displays, different effects could occur (for example, with the cube shape used in Experiment 1). Rather, the point was to investigate judgments in isolation and then to compare such individual results to known effects on more complex images. Only two perspective $(r, f)$ values were used: $\left[\left(r_{1}, f_{1}\right)=(27,13.5),\left(r_{2}, f_{2}\right)=\right.$ $(108,13.5) \mathrm{cm}]$ in both distance and angle estimation tasks.

For both tasks, central (object centered at the origin, Figure 1) and eccentric (object centered $12 \mathrm{~cm}$ to the right of the origin on the $\mathrm{x}$-axis) projections were used. In addition, two rotation types were employed-rotations about the $y$-axis alone and rotations about the yz-axes simultaneously. The rotation speed was constant at $1 \mathrm{~Hz}$, and subjects observed each condition three times with 4-sec exposure time. Three different angles of the rotating line were used $(0,45$, and $60 \mathrm{deg}$ in the $x y$ plane), and three intersection angles of 30,45 , and 90 deg were used in the angle-estimation study (see Figure 5), one line fixed at $30 \mathrm{deg}$ in the xy plane.

Procedure. The subjects were seated $1 \mathrm{~m}$ from the viewing screen and told they would see an object moving on the screen (Figure 3). When the motion was complete, they were to respond as to whether they saw the motion as rigid or plastic (bending or object changing in shape) and as to whether it was two-dimensional or threedimensional; they were also to estimate angles or lengths. These latter judgments were made by either adjusting the length of an extendable slit or the angle between two slits shown in Figure 5. Viewing and judgments were always made with the dominant eye.

For each of the length and angle estimation tasks, observers received 72 trials: perspectives $(2) \times$ (central, eccentric: 2$) \times$ rotations (2) $\times$ angle (3) $\times$ replications (3). Here, as in all these experiments, only few replications were employed so as to avoid possible development of strategies based on familiarization with the specific configurations.

\section{Results}

Figure 6a illustrates the percentage of plasticity reports for both tasks as a function of the experimental treatments, while Figure $6 \mathrm{~b}$ shows the length and angle estimations over observers.

No observable differences were found over length estimates. However, a four-way repeated measures analysis of variance (perspective $x$ rotation $\times$ angle
$X$ center $\times$ subjects) yielded significant effects of all conditions (and interactions) on angle-estimation errors $(p<.05)$. These estimation data include length and angle estimates for configurations independent of their perceived rigidity. The reason for including both responses was simply because it is not necessarily the case that the rigidity judgment is contingent on clear and distinct estimates of length and angle. In an objective euclidean space, fixed lengths and angles presume a rigid object, and vice versa. However, this is not true here. For example, observers were very consistent about the length of the line, although variations in its rigidity were quite high, particularly with nonparallel, two-parameter rotations and eccentric views.

As the perspective view and transformation types departed from the central parallel projection of an object centrally rotating about the y-axis, the perceived rigidity (hence invariance under euclidean motions) decreased. Also, perceived length was more stable than perceived angle within the various experimental manipulations employed. Angle is a relational structure involving, at least, the (local) comparison of two contiguous lines, and in a more strict geo-
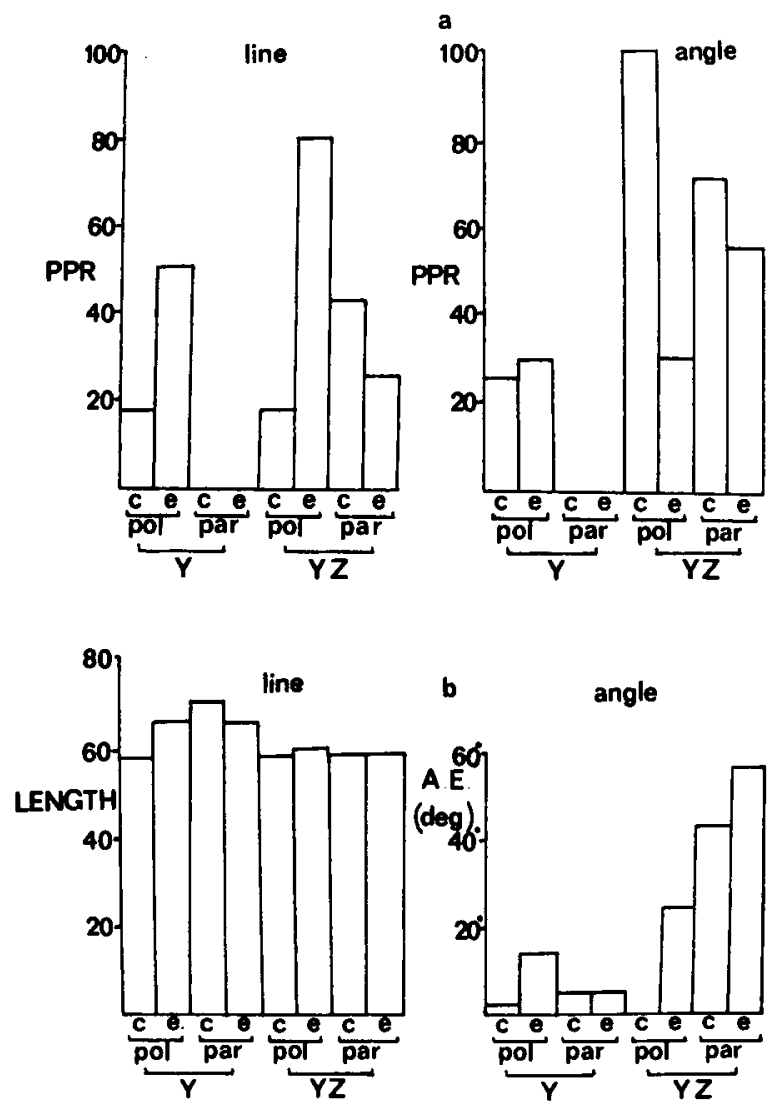

Figure 6. (a) Percentage of plasticity responses (PPR) over perspective type $(\mathrm{pol}=$ polar, par $=$ parallel $)$, rotation center $(c=$ center, $e=$ eccentric), rotation type ( $y$-axis, yz-axes) for line and angle stimuli. (b) Length estimation (1 unit $=.25 \mathrm{~cm}$ ) and angle estimation (AE: deg) for experimental conditions. 
metric sense, their lengths (when triangles are of concern). In conjunction with the results of the previous experiment, the stability of length judgments of such simple 3D objects is consistent with observers' ability to set their own reference frames for corresponding perspective views. However, observers have considerable problems with extracting 3D properties of rigid motion and stable angle estimations with these two line images.

These data suggest that, at least within the context of the KDE, the "perceptual complementarity" or correspondence between perceived and physical transformations holds for reference frames, simple length estimations, but does not hold with the first level of relational information-angles-particularly as the object departs from a parallel, centered, single $y$-axis rotation. Just how much these results are a function of the displays used is the subject of the following experiment, in which an attempt was made to investigate how our perceptual reconstructions within this context are invariant of the perspective view and are identifiable with real 3D objects.

\section{EXPERIMENT 3: PERSPECTIVE PREFERENCE AND OBJECT CORRESPONDENCE}

The results of Experiments 1 and 2 indicate that, to a large extent, our "view" of 3D space from its 2D projections (in the context of the KDE) is determined by the perspective information, per se, and the intrinsic geometry used to generate the percept of a depth space. Although the basic properties of perspective view, angle, length, and perceived rigidity were examined above, the ability to recognize a 3D object under these conditions has not been studied or related to these results. In this experiment we have studied the ability to identify such objects.

\footnotetext{
Method

Subjects. The subjects were six graduate students at the University of Newcastle with no known visual defects.

Stimulus and Apparatus. Two stimulus objects (a square and cube) were computer generated and displayed under identical KDE conditions, as in the previous experiment, except for the use of only three perspective values with linear perspectives ( $L_{p}$ values) of $.3, .7, .8$ (Figure 7 ). These objects were then rotated about the $y-, x y-$, and $y z-a x e s$, resulting in 2 (objects) $\times 3$ (perspective) $\times$ 3 (rotation type) $=18$ display conditions. Wire objects were composed with linear perspectives ${ }^{3}(4)$ of: $.2, .3, .5, .7,1.0$ for the square and $.2, .3, .5, .7, .9,1.0$ for the cube (Figure 7 ). One side of the square ("nearest") and face of the cube were $10 \mathrm{~cm}$ and $10 \times 10 \mathrm{~cm}$, respectively, the "farthest" (smaller) elements being determined by the perspective value.

Procedure. A trial consisted of the observer's monocularly viewing a particular rotating object for a period of $4 \mathrm{sec}$ through the viewing box used in Experiment 1 . As in previous KDE studies, the observer was to respond as to whether the object was rigid or elastic and $2 \mathrm{D}$ or $3 \mathrm{D}$. After making these judgments, the subject was asked to rate the wire objects as to their similarity to what had been perceived and to rotate the most similar object according to how the motion had been perceived. After 10 training trials and the subject felt comfortable with the task, he or she received 10
}

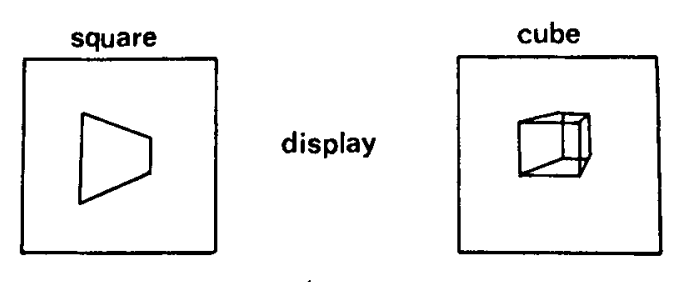

wire

alternatives
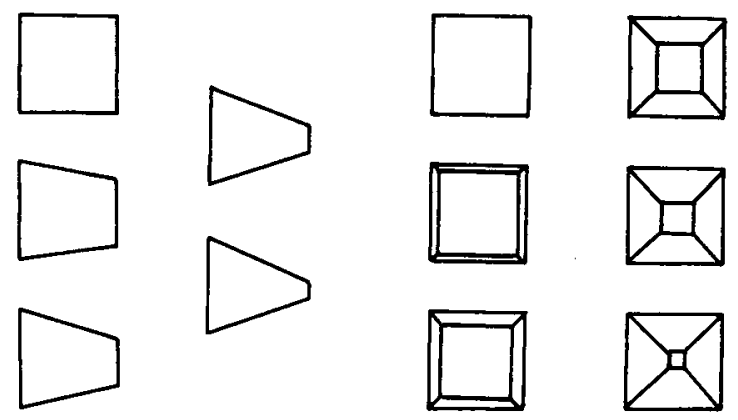

Figure 7. Displayed stimulus characteristics and wire objects used in Experiment 3.

trials for each condition (180 in all per subject), allocated randomly over all trials.

\section{Results}

Figures 8 and 9 illustrate results for the two objects as a function of the perspective and rotation variables.

Analyses of variance on rigidity and veridical motion reports [object $(2) \times$ perspective $(3) \times$ rotation type $(3) \times$ subjects] yielded significant effects of rotation type and perspective view in both cases [rigidity and perspective, $F(2,10)=29.82, p<.01$; rotation, $\mathrm{F}(2,10)=13.97, \mathrm{p}<.01$; veridical motion and perspective, $F(2,10)=24.39, \mathrm{p}<.01$; rotation, $F(2,10)$ $=34.57, \mathrm{p}<.01]$.

Additional analyses of variance were computed on first preferences for the wire objects as a function of rotation type and perspective (rotation $\times$ perspective $x$ object $x$ subjects). Results indicated significant perspective [square, $F(2,10)=15.0, p<0.01$; cube, $F(2,10)=13.99, p<.01$ ], object perspective [square, $\mathrm{F}(4,20)=140.41, \mathrm{p}<.01$; cube, $\mathrm{F}(5,25)=135.0$, $\mathrm{p}<.01$ ], and rotation [square, $\mathrm{F}(2,10)=7.89, \mathrm{p}<.01$; cube, $F(2,10)=5.07, p<.01]$ effects.

These data illustrate the difficulty observers have identifying the "true" 3D nature (although the 3D objects were squares and cubes $-L_{p}=1.0$ - the observers were not told so) and motion of objects, particularly when viewed from perspectives and rotations which depart significantly from the parallel, central view with only one-parameter rotations. Of specific interest here is the discrepancy between object identification and rotation detection. That is, although observers could accurately describe the mo- 


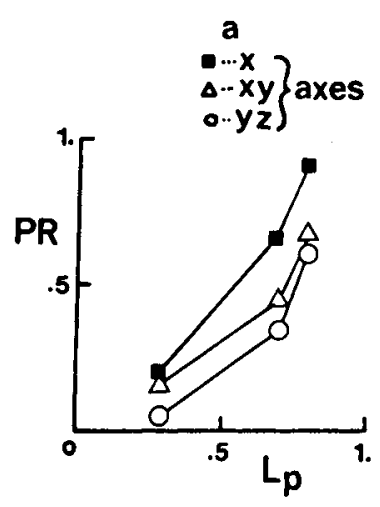

square
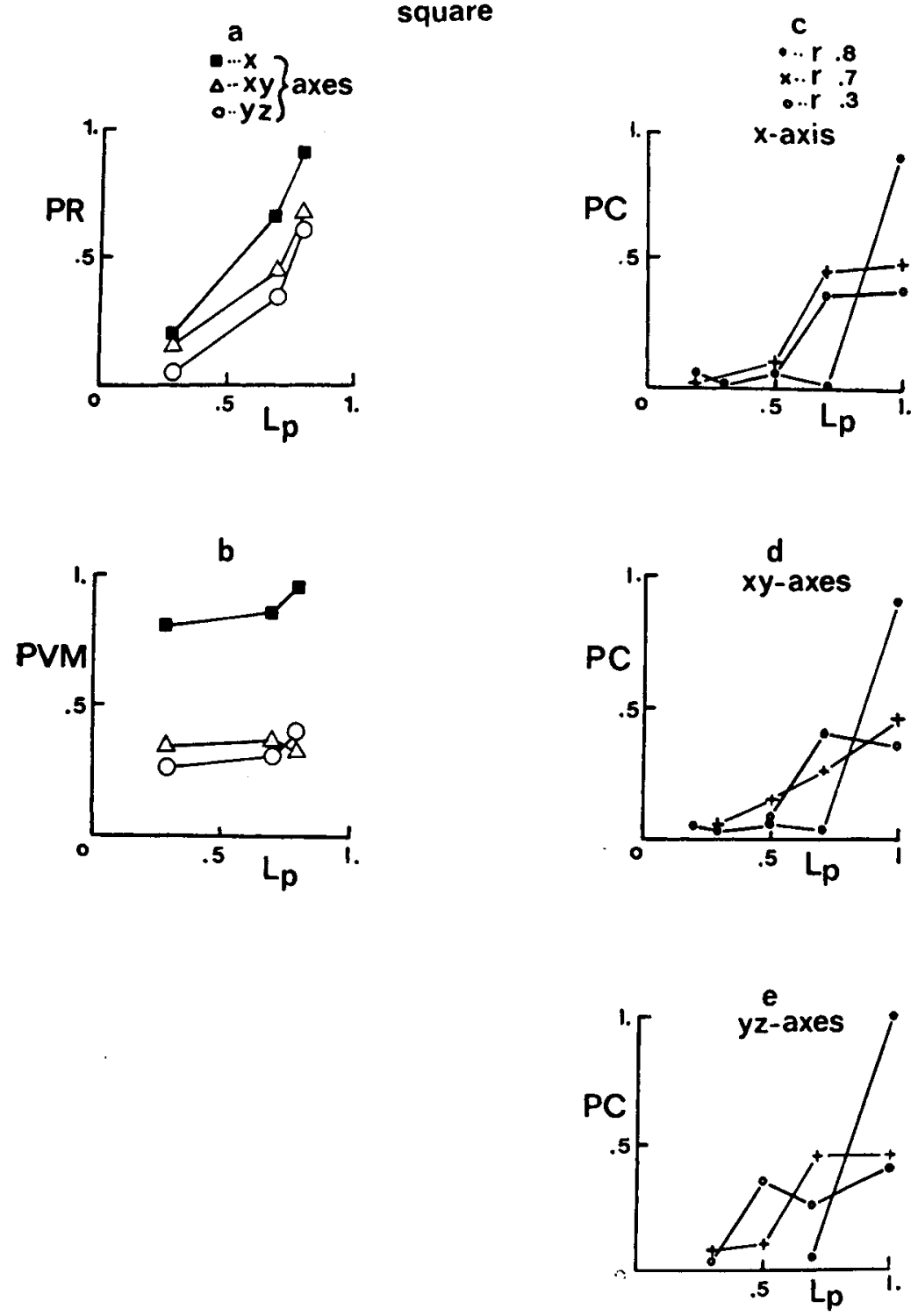

Figure 8. Proportion of (a) rigidity (PR) and (b) veridical motion reports (PVM) for the cube stimulus as a function of perspective $\left(I_{p}\right.$ values). (c-e) Percentage of times (PC) wire cubes were rated as similar (each wire cube defined by an $L_{p}$ value) over rotation types.

tion path in the simple rotation about the y-axis, they could not estimate its nature ${ }^{4}$ consistently and independently of the perspective view. In addition, when more complex motions were used (xy-, yz-axes rotations), the object's motion, which was accompanied by a loss of perceived object rigidity, was considerably more difficult to describe. This was also the case in the previous experiment in which the angular stimulus was particularly difficult to perceive as rigid.

\section{DISCUSSION}

The results delimit the domain of "perceptual complementarity" by indicating the types of per- spective conditions, subjective estimation tasks, and percepts which underlie the reconstruction process, at least in the KDE. In the three experiments reported, data were collected on: (1) the ability of observers to adjust their perspective view as a function of the $2 \mathrm{D}$ perspective information given; (2) the ability of observers to extract length and angle information as a function of perspective, rotational and eccentricity components of the object; and, (3) the ability of observers to identify an object and its motion under various perspective and rotational conditions.

Although a variety of stimulus objects were used, the results across the three experiments combine to depict a limited geometry for the reconstruction pro- 

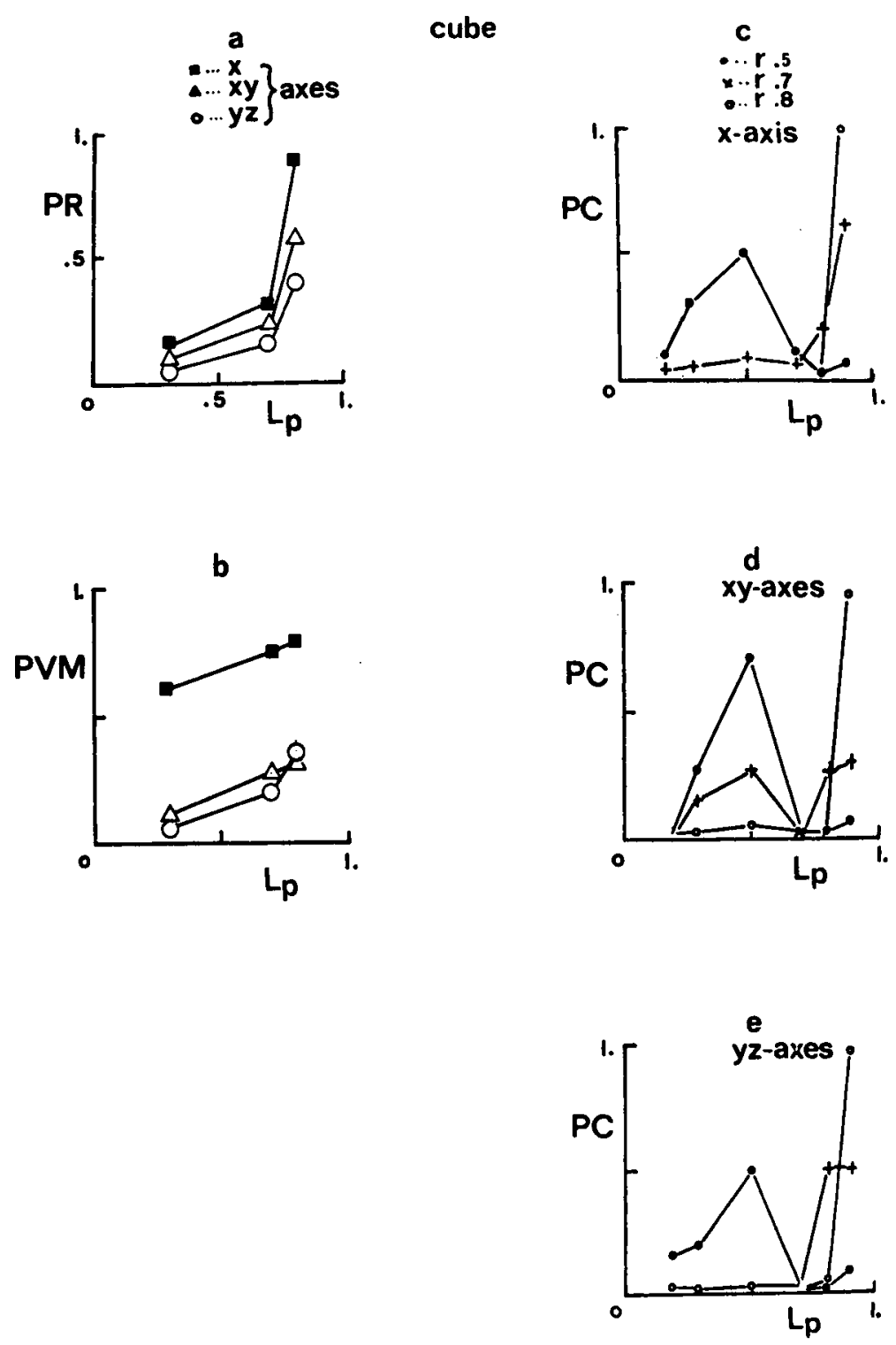

Figure 9. Portion of (a) rigidity (PR) and (b) veridical motion reports (PVM) for the cube stimulus as a function of perspective ( $L_{p}$ values). (c-e) Percentage of times (PC) wire cubes were rated as most similar (each wire cube defined by an $\mathbf{L}_{p}$ value) over rotation types.

cess. The results, particularly those for angle estimation in Experiment 1 and object identity in Experiment 3 , indicate that the perspective information (that is, the relative lengths of lines on the 2D projection surface) is, in various degrees, confused with the underlying 3D object.

Traditional studies of the KDE have centered on percepts of $3 \mathrm{D}$ rotations or oscillations and have generally not varied the projection geometry or observed how subjects perceive the distances, lengths, angles, motion paths, and object identities studied here. For this reason, these data cannot be directly compared with past results except to the extent that they demonstrate, as with past results (Caelli, 1981a; Wallach
\& O'Connell, 1953), that our abilities to reconstruct 3D objects are limited to a spatiotemporal "window." This window corresponds to linear perspectives greater than about .7 , since at or above this value subjects could readily extract the cube or square structure (that is, the wire object with $L_{p}=1.0$ ). Furthermore, consistent across all the tasks and stimuli used, the only rotation which produced percepts highly consistent with the physical states was the oneparameter $y$-axis rotation of an object centered about the origin. Considered in the light of the temporal limitation (that the effect significantly declines above $2 \mathrm{~Hz}$, zero at about $4 \mathrm{~Hz}$ ) for the KDE already investigated by Caelli (1980), these data restrict the 
reconstruction process to a very small combination of spatiotemporal states.

Standards for optimizing the 3D effects can be established from these data. For example, (1) the viewing distance should be at least six times more than the projection plane distance $\left(r \geqslant 6 f\right.$, from $L_{p} \geqslant .7$ ), (2) eccentric projections should be less than an equivalent of 6 deg of visual angle (from the loss of consistent estimations in Experiment 2 with the 12-cm eccentricity), and (3) only single parameter rotations of less than $2 \mathrm{~Hz}$ should be employed.

Shepard's (1981) concept of "perceptual complementarity," in the context of apparent motion and mental rotations, deals with the ability of observers to perform mental transformations consistent with rigid euclidean motions of objects. In a more direct way, the $\mathrm{KDE}$ involves the extraction of rigid motions from projection information. Whether equivalent restrictions occur with the mental rotation task (MRT), as have been found here, is a subject for further experimentation. However, it should be noted that the actual stimulus configurations used probably affect what is perceived. In an experiment on the effects of perspective and rotation type on the MRT with compound cube stimuli, we have found that observers' RT are not significantly affected by such parameters. We do not know is these stable results are due to the use of such compound spatial information (presumably additive in effect) or whether the MRT involves cognitive processes that are less dependent on perspective information than the KDE is.

In a similar way, the major difficulty with the experiments reported here is that they each involved different images as well as tasks. The reason for this is clear: the aim in each experiment was to isolate and measure different aspects of the reconstruction process. However, in doing so, comparisons across tasks must be qualified. For example, loss of rigidity and object identity in Experiment 3 must relate to loss of angle and length information, as measured in Experiment 2 , but since no equivalent to a spatial summation experiment has yet been performed, no exact comparison is possible. That is, can a given object's reconstruction properties (rigidity, angles, etc.) be predicted from the reconstruction properties of isolated segments which compose the object?

Although object identification, motion paths, and angle perception were all significantly affected by the perspective views involved, length and distance infor- mation were preserved (at least up to a linear transformation). Results from Experiments 1 and 2 indicate that the observer can reorganize his or her reference frame to fit in with the perspective view used, and that, under such circumstances, judgments of the perspective distance parameters $(\mathrm{r}, \mathrm{f})$ and intrinsic object lengths are consistent with (or complementary to) the objective states, even if they do not match his or her own physical location. Consequently, it must be the (internal) object relational information (angles, relative lengths, etc.) which presents the major problem to the reconstruction process. The proximity and filter characteristics for the extraction of this latter information is the subject of a recent paper by Caelli (1981b).

\section{REFERENCES}

Braden, J. The effects of varying linear perspective, movement parallax, and speed of rotation on perceived oscillation of twodimensional trapezoidal stimuli. Perception \& Psychophysics, 1978, 23, 51-57.

CaElli, T. M. Amplitude, frequency and phase determinants of perceived rotations and rigidity in the kinetic depth effect. Biological Cybernetics, 1980, 36, 213-219.

CAELli, T. M. Visual perception. Theory and practice. Oxford: Pergamon Press, 1981. (a)

CaElli, T. M. On the spatio-temporal determinants of some motion effects. Acta Psychologica, 1981, 48, 175-185. (b)

Caelli, T. M., Hoffman, W. C., \& Lindman, H. Subjective Lorentz transformations and the perception of motion. Journal of the Optical Society of America, 1978, 68, 402-417.

Gilinsky, A. Perceived size and distance in visual space. Psychological Review, 1951, 58, 460-482.

RoBinson, J. The psychology of visual illusions. London: Hutchinson, 1972.

She Pard, R. Psychophysical complementarity. In M. Kubovy \& J. Pomerantz (Eds.), Perceptual organization. Hillsdale, N.J: Erlbaum, 1981.

Wallach, H., \& O'Connell, D. N. The kinetic depth effect. Journal of Experimental Psychology, 1953, 45, 205-217.

\section{NOTES}

1. Any two rotation axes are sufficient to define a threedimensional rotation.

2. The phase angle between two rotating elements refers to the angular difference on the plane of rotation with respect to the rotation axis.

3. That is, the ratios of the back/front side (square) or surface (cube) extents.

4. The object properties including lengths, angles, etc.

(Manuscript received February 3, 1981; revision accepted for publication January 5,1982 .) 